\title{
Routine childhood vaccinations did not increase the risk of incident type 1 diabetes in Danish children
}

Hviid A, Stellfeld M, Wohlfahrt J, et al. Childhood vaccination and type 1 diabetes. N Engl J Med 2004;350:1398-404.

Are routinely administered childhood vaccines associated with an increased incidence of type 1 diabetes mellitus in a cohort of Danish children?

\section{METHODS}

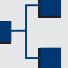

Design: a cohort study of children followed up from birth to a mean age of 6.4 years.

(19)

Setting: Denmark.

1.

Participants: 739694 children born in Denmark from January 1990 to December 2000

$\Lambda$

Risk factors: vaccinations with any of 6 groups of vaccines including Haemophilus influenza type b; diphtheria, tetanus, and inactivated poliovirus; diphtheria, tetanus, acellular pertussis, and inactivated poliovirus; whole cell pertussis; measles, mumps, and rubella; and oral poliovirus (data from vaccination reports of the National Board of Health). Sensitivity analyses considered the presence of a sibling with type 1 diabetes as a predisposing factor

1 Outcomes: incidence of type 1 diabetes (from January 1990 to December 2001) (data from the Danish National Hospital Register).

\section{MAIN RESULTS}

The incidence of type 1 diabetes was 0.14 cases/1000 person-years among all children, and 6.2 cases/1000 person-years among those who had a sibling with type 1 diabetes. Routine childhood vaccinations were not associated with an increased incidence of type I diabetes mellitus among all children or among those who had a sibling with type 1 diabetes (table).

For correspondence: Mr A Hviid, Danish Epidemiology Science Centre, Statens Serum Institut, Copenhagen, Denmark. aii@ssi.dk

Sources of funding: Danish National Research Foundation and the Danish Medical Research Council.

\section{CONCLUSION}

Routinely administered childhood vaccines were not associated with an increased incidence of type 1 diabetes mellitus in a cohort of Danish children

\section{Commentary}

Trer he study by Hviid et al contributes new evidence refuting a correlation between childhood immunisations and development of type 1 diabetes. Although some studies have suggested a link, ${ }^{1-3}$ no other researchers have verified this, and recent reports and studies reject this theory. ${ }^{4} 5$

The size of the cohort and the length of follow up add strength to the study conclusion. The authors estimated rate ratios, a statistical analysis that allows comparison of the incidence rates of a condition in 2 groups. In this case, the groups were defined according to vaccination status, which was considered to change over time, so that children in the study contributed person-years as both unvaccinated and vaccinated participants.

In addition, the authors considered whether a trend was associated with the number of vaccination doses. Children who had siblings with type 1 diabetes were considered as a subgroup because genetic susceptibility to type 1 diabetes is a well documented risk factor. ${ }^{67}$

Although vaccination schedules vary among countries, the findings of this and other studies ${ }^{45}$ are relevant to all nurses providing advice to parents about childhood immunisation, particularly families where a strong genetic tendency to diabetes already exists. These families should be counselled that current evidence does not support a causal link between childhood vaccination and type 1 diabetes. Judith Carrier, RGN, MSc, PGCE, Sp practitioner(PN) School of Nursing and Midwifery Studies Cardiff University

Classen DC Classen JB. Infect Dis Clin Pract 1997:6:449-54. Wales, UK

2 Classen JB. Autoimmunity 1996;24:137-45.

3 Classen JB, Classen DC. Autoimmunity 2002;35:247-53

4 Institute of Medicine. Immunizations safety review: multiple immunizations and immune dysfunction (2002). hittp://www.nap.edu/books/ $0309083281 / \mathrm{html} /$ index. htm

5 DeStefano F, Mullooly JP, Okoro CA, et al. Pediatrics 2001;108:E112. 6 Cox NJ, Wapelhorst B, Morrison VA, et al. Am J Hum Genet 2001;69:82030.

7 Diabetes UK. Research features: genetic research. Updated Feb 2003. http://www. diabetes.org.uk/research/feature/reshome

Association between routinely administered childhood vaccines and incident type 1 diabetes mellitus in Danish children at a mean age of 6.4 years*

\begin{tabular}{lll}
\hline & & Rate ratio (95\% Cl) \\
\cline { 2 - 3 } Vaccine & All childrent & Children with $\geqslant 1$ sibling with type 1 diabetes $¥$ \\
\hline Haemophilus influenza type b & $0.91(0.74$ to 1.12$)$ & $1.38(0.58$ to 3.31$)$ \\
Diphtheria, tetanus, and inactivated poliovirus & $1.02(0.75$ to 1.37$)$ & $3.03(0.41$ to 22.63$)$ \\
Diphtheria, tetanus, acellular pertussis, and inactivated poliovirus & $0.96(0.71$ to 1.30$)$ & $1.36(0.50$ to 3.70 \\
Whole cell pertussis & $1.06(0.80$ to 1.40$)$ & $1.68(0.39$ to 7.19$)$ \\
Measles, mumps, and rubella & $1.14(0.90$ to 1.45$)$ & $0.86(0.34$ to 2.14$)$ \\
Oral poliovirus & $1.08(0.74$ to 1.57$)$ & $2.01(0.46$ to 8.71$)$ \\
\hline
\end{tabular}

${ }^{*}$ Rate ratios (comparing rates in children vaccinated with $\geqslant 1$ dose $v$ unvaccinated) show that all associations are not significant; $\mathrm{Cl}$ defined in glossary.

tRate ratios adjusted for age, calendar period, and sex.

†Rate ratios adjusted for age, calendar period, sex, and number of siblings. 